\title{
A New Material Sensitivity Analysis for Electromagnetic Inverse Problems
}

\author{
Jin-Kyu Byun ${ }^{1}$, Hyang-Beom Lee ${ }^{1}$, Hyeong-Seok Kim² ${ }^{2}$ and Dong-Hun Kim ${ }^{3 *}$ \\ ${ }^{1}$ School of Electrical Engineering, Soongsil University, Seoul 156-743, Korea \\ ${ }^{2}$ School of Electronic Engineering, Chung-Ang University, Seoul 156-756, Korea \\ ${ }^{3}$ School of Electrical Eng. \& Computer Sci., Kyungpook Nat'l. Univ., Daegu 702-701, Korea
}

(Received 27 August 2010, Received in final form 27 October 2010, Accepted 3 November 2010)

\begin{abstract}
This paper presents a new self-adjoint material sensitivity formulation for optimal designs and inverse problems in the high frequency domain. The proposed method is based on the continuum approach using the augmented Lagrangian method. Using the self-adjoint formulation, there is no need to solve the adjoint system additionally when the goal function is a function of the $S$-parameter. In addition, the algorithm is more general than most previous approaches because it is independent of specific analysis methods or gridding techniques, thereby enabling the use of commercial EM simulators and various custom solvers. For verification, the method was applied to the several numerical examples of dielectric material reconstruction problems in the high frequency domain, and the results were compared with those calculated using the conventional method.
\end{abstract}

Keywords : material sensitivity, inverse problems, self-adjoint formulation, sensitivity analysis

\section{Introduction}

Since the early 1990s, sensitivity analysis using the adjoint variable method (AVM) has been used widely for electromagnetic optimal designs and inverse problems in the low frequency domain [1-4]. However, in the high frequency domain, most sensitivity analyses of electromagnetic problems have been based on analytic sensitivity calculations with a small number of design parameters $[5,6]$.

Recently, increasing effort has been made to introduce AVM to optimization and inverse problems in the high frequency domain. AVM allows rapid and efficient calculations of the sensitivity when there are a large number of design parameters. This makes it particularly useful for high-resolution inverse problems, where stochastic methods, such as genetic algorithms, particle swarm optimization (PSO), etc., are computationally prohibitive [7]. Previous studies gave a few examples, where a dielectric waveguide filter [8] and microstrip low-pass filter (LPF) with a defected ground structure [9] were optimized utilizing the AVM.

The essence of the AVM is the construction of an

*Corresponding author: Tel: +82-53-950-5603

Fax: +82-53-950-5603, e-mail: dh29kim@ee.knu.ac.kr adjoint system using an adjoint source, which is obtained by differentiating the goal function with respect to the state variables (electric or magnetic field). The normal procedure for the AVM is to solve this adjoint system and obtain adjoint variables that are used in the sensitivity equation along with the solution of the primary system. The AVM already has a huge advantage over the finitedifference method (FDM), where the system must be solved $n p$ (number of design parameters) times because the entire sensitivity vector can be calculated with only one additional solving of the adjoint system. Moreover, since the adjoint system is often interpreted as a dual system of the primary system and has the same characteristics as the primary one except for the source term (excitation), it can be solved with less computational burden than the primary one when a custom solver, such as LU decomposition, is used [1].

Many studies have been carried out to remove this additional step and calculate the adjoint variable directly from the state variable solution of the primary system. In the low-frequency domain, using the self-energy formulation derived from the mutual energy, the sensitivity can be calculated from a solution of the primary system alone if the objective function is defined in terms of the self flux-linkage in the source coil [2]. With high frequency systems, Nikolova et al. presented a time-domain formu- 
lation for the self-adjoint $S$-parameter sensitivity with respect to the material interface and boundary interface in structured grids based on a discrete approach that does not require a solution of the adjoint system [10]. Recently, they expanded their approach to the frequency-domain formulation [11]. However, their formulation is based on differentiating the finite-difference frequency-domain (FDFD) system matrices and regular grids, where the sensitivity solver is generated on its own. Naturally, it is most suited for problems that are already discretized with structured grids.

This paper proposes a new, more general frequencydomain self-adjoint formula for the $S$-parameter sensitivity calculation with respect to the material properties. This method is based on recently derived continuum sensitivity analysis for high frequency systems, which employs the direct differentiation of the continuous EM governing equation before discretization. This approach is independent of the specific analysis method or gridding technique, and commercial EM simulators as well as custom solvers can be used for optimization and inverse problems. The proposed method was applied to the several numerical examples of dielectric material reconstruction problems inside a parallel plate waveguide to assess its feasibility and effectiveness.

\section{Self-Adjoint Material Sensitivity Formulation}

\subsection{General material sensitivity formulation based on continuum approach}

This section reviews the general material sensitivity formulation for high frequency electromagnetic systems. Fig. 1 shows an exemplary 2-port system (rectangular waveguide) with a dielectric discontinuity inside analysis domain $\Omega$ Ports 1 and 2 are defined on boundaries $\Gamma_{1}$ and $\Gamma_{2}$.

The scattering parameters ( $S$-parameters) are defined in relation to the incident and reflected fields at the ports [12]. For example, $S_{11}$ can be defined as

$$
S_{11}=\frac{\mathbf{H}_{1}^{\text {ref }}}{\mathbf{H}_{1}^{\text {inc }}}=\frac{\mathbf{H}_{1}^{\text {tot }}-\mathbf{H}_{1}^{\text {inc }}}{\mathbf{H}_{1}^{\text {inc }}}
$$

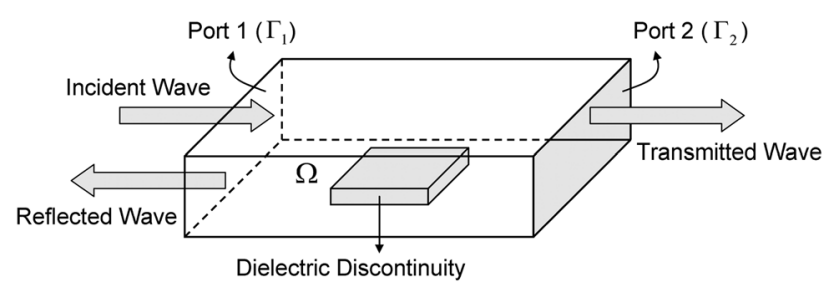

Fig. 1. Rectangular waveguide with a dielectric discontinuity. where $\mathbf{H}_{1}^{\text {inc }}, \mathbf{H}_{1}^{\text {ref }}$, and $\mathbf{H}_{1}^{\text {tot }}$ are the incident, reflected, and total magnetic fields at port 1, respectively. $S_{11}$ is a linear function of the total magnetic field $\mathbf{H}_{1}^{\text {tot }}$ at port 1 because the incident field $\mathbf{H}_{1}^{i n c}$ is a known vector. For simplicity, the total magnetic field $\mathbf{H}^{\text {tot }}$ is written without the superscript as $\mathbf{H}$ from now on.

Assume that an objective function $O$ is a function of $S_{11}$. Since $S_{11}$ is a function of $\mathbf{H}$ on $\Gamma_{1}$, as shown in (1), $O$ can be defined on boundary $\Gamma_{1}$ as follows:

$$
O=\int_{\Gamma_{1}} g(\mathbf{H}(\mathbf{p})) d \Gamma
$$

where $g$ is a scalar function of $\mathbf{H}$, and $\mathbf{p}$ is a system parameter vector that controls the permittivity $\varepsilon$ or permeability $\mu$ distribution of the system. In the time-harmonic case, the governing equation is given by the vector wave equation for $\mathbf{H}$ as

$$
\nabla \times\left(\varepsilon_{r}^{-1} \nabla \times \mathbf{H}\right)-k_{0}^{2} \mu_{r} \mathbf{H}=\mathbf{0}
$$

where $k_{0}$ is the wavenumber in free space. On $\Gamma_{1}$, where incident field is imposed, mixed boundary condition should be defined, which may be expressed as [13],

$$
\varepsilon_{r}^{-1} \hat{n} \times(\nabla \times \mathbf{H})+\gamma_{h} \hat{n} \times(\hat{n} \times \mathbf{H})=\mathbf{V}
$$

where $\hat{n}$ is a unit vector pointing outward normal to the boundary $\Gamma_{1}, \gamma_{h}$ is a known parameter and $\mathbf{V}$ denotes a known vector with a magnitude proportional to that of the incident field $\mathbf{H}_{1}^{i n c}$.

The augmented Lagrangian method was used to deduce the sensitivity formula [4]. First, the augmented objective function $\bar{O}$ was developed by adding the variational form of the vector wave equation (3) to (2) as

$$
\bar{O}=\int_{\Gamma_{1}} g d \Gamma+\int_{\Omega} \lambda \cdot\left[\nabla \times\left(\varepsilon_{r}^{-1} \nabla \times \mathbf{H}\right)-k_{0}^{2} \mu_{r} \mathbf{H}\right] d \Omega
$$

where $\lambda$ is an arbitrary Lagrange multiplier vector. To simplify the final form of the sensitivity equation, a specific condition was imposed on $\lambda$ later in the derivation, which allows $\lambda$ to be interpreted as the adjoint variable vector. Applying the first vector Green's theorem to (5), we obtain

$$
\begin{aligned}
\bar{O}= & \int_{\Gamma_{1}} g d \Gamma+\int_{\Omega}\left[\varepsilon_{r}^{-1}(\nabla \times \lambda) \cdot(\nabla \times \mathbf{H})-k_{0}^{2} \mu_{r} \lambda \cdot \mathbf{H}\right] d \Omega \\
& +\int_{\Gamma} \varepsilon_{r}^{-1} \lambda \cdot(\hat{n} \times \nabla \times \mathbf{H}) d \Gamma .
\end{aligned}
$$

The only nonzero contribution of the second surface integral comes from $\Gamma_{1}$. Substituting (4) into (6), produces

$$
\begin{aligned}
\bar{O}= & \int_{\Gamma_{1}} g d \Gamma+\int_{\Omega}\left[\varepsilon_{r}^{-1}(\nabla \times \lambda) \cdot(\nabla \times \mathbf{H})-k_{0}^{2} \mu_{r} \lambda \cdot \mathbf{H}\right] d \Omega \\
& +\int_{\Gamma_{1}}\left[\gamma_{h}(\hat{n} \times \lambda) \cdot(\hat{n} \times \mathbf{H})+\lambda \cdot \mathbf{V}\right] \mathrm{d} \Gamma .
\end{aligned}
$$


By taking the variation of both sides of (7) with respect to small changes, $\delta \mathbf{p}$, in the system parameters, the first variation of the augmented objective function $\delta \bar{O}$ can be developed as follows:

$$
\begin{aligned}
& \delta \bar{O}=\int_{\Gamma_{1}}\left[\frac{\partial g}{\partial \mathbf{H}} \cdot\left(\delta \mathbf{H}+\frac{\partial \mathbf{H}}{\partial \mathbf{p}} \delta \mathbf{p}\right)\right] d \Gamma \\
& +\int_{\Omega}\left[\varepsilon_{r}^{-1}(\nabla \times \lambda) \cdot\left\{\nabla \times\left(\delta \mathbf{H}+\frac{\partial \mathbf{H}}{\partial \mathbf{p}} \delta \mathbf{p}\right)\right\}+\frac{\partial\left(\varepsilon_{r}^{-1}\right)}{\partial \mathbf{p}}(\nabla \times \lambda) \cdot(\nabla \times \mathbf{H}) \delta \mathbf{p}\right] d \Omega \\
& -\int_{\Omega} k_{0}^{2}\left[\mu_{r} \lambda \cdot\left(\delta \mathbf{H}+\frac{\partial \mathbf{H}}{\partial \mathbf{p}} \delta \mathbf{p}\right)+\frac{\partial \boldsymbol{\mu}_{r}}{\partial \mathbf{p}} \lambda \cdot \mathbf{H} \delta \mathbf{p}\right] d \Omega \\
& +\int_{\Gamma_{1}}\left[\gamma_{h}(\hat{n} \times \lambda) \cdot\left\{\hat{n} \times\left(\delta \mathbf{H}+\frac{\partial \mathbf{H}}{\partial \mathbf{p}} \delta \mathbf{p}\right)\right\}\right] d \Gamma .
\end{aligned}
$$

Using the notations $\mathbf{g}_{\mathbf{H}} \equiv \partial g / \partial \mathbf{H}$ and $\bar{\lambda} \equiv(\delta \mathbf{H}+(\partial \mathbf{H} / \partial \mathbf{p}) \delta \mathbf{p})$, (8) can be reduced to,

$$
\begin{gathered}
\delta \bar{O}=\int_{\Gamma_{1}}\left(\mathbf{g}_{\mathbf{H}} \cdot \bar{\lambda}\right) d \Gamma+\int_{\Omega}\left[\varepsilon_{r}^{-1}(\nabla \times \lambda) \cdot(\nabla \times \bar{\lambda})+\frac{\partial\left(\varepsilon_{r}^{-1}\right)}{\partial \mathbf{p}}(\nabla \times \lambda) \cdot(\nabla \times \mathbf{H}) \delta \mathbf{p}\right] d \Omega \\
-\int_{\Omega} k_{0}^{2}\left[\mu_{r} \lambda \cdot \bar{\lambda} \frac{\partial \mu_{r}}{\partial \mathbf{p}} \lambda \cdot \mathbf{H} \delta \mathbf{p}\right] d \Omega+\int_{\Gamma_{1}} \gamma_{h}(\hat{n} \times \lambda) \cdot(\hat{n} \times \bar{\lambda}) d \Gamma
\end{gathered}
$$

where $\bar{\lambda}$ is an arbitrary vector that corresponds to the first variation of the magnetic field. The following condition was imposed on the Lagrange multiplier vector $\lambda$ to simplify the final form of the sensitivity:

$$
\begin{aligned}
& \int_{\Omega}\left[\varepsilon_{r}^{-1}(\nabla \times \bar{\lambda}) \cdot(\nabla \times \lambda)-k_{0}^{2} \mu_{r} \bar{\lambda} \cdot \lambda\right] d \Omega \\
& \quad+\int_{\Gamma_{1}}\left[\gamma_{h}(\hat{n} \times \lambda) \cdot(\hat{n} \times \lambda)+\lambda \cdot \mathbf{g}_{\mathbf{H}}\right] d \Gamma=0
\end{aligned}
$$

which can be interpreted as a variational form of the adjoint system. A comparison of (10) with the second and third term on the right-hand side of (7) shows that the only difference between the primary and adjoint system is the source term ( $\mathbf{V}$ for primary system and $\mathbf{g}_{\mathbf{H}}$ for adjoint one) on the mixed boundary $\Gamma_{1}$. Therefore, the adjoint system can be solved by modifying the source of the primary system from $\mathbf{V}$ to $\mathbf{g}_{\mathbf{H}}$. Substituting (10) into (9), and dividing both sides with $\delta \mathbf{p}$, the material sensitivity formula in high frequency system can be derived as follows:

$$
\frac{d \bar{O}}{d \mathbf{p}}=\int_{\Omega}\left[\frac{\partial\left(\varepsilon_{r}^{-1}\right)}{\partial \mathbf{p}}(\nabla \times \mathbf{H}) \cdot(\nabla \times \lambda)-k_{0}^{2} \frac{\partial \mu_{r}}{\partial \mathbf{p}} \mathbf{H} \cdot \lambda\right] d \Omega .
$$

In summary, the material sensitivity of the augmented objective function $\bar{O}$ with respect to the system parameter vector $\mathbf{p}$ can be calculated in the following steps:

(a) Solve the original (primary) electromagnetic system (3) and obtain a field solution $\mathbf{H}$.

(b) Calculate the adjoint source $\mathbf{g}_{\mathbf{H}} \equiv \partial g / \partial \mathbf{H}$.

(c) Solve the adjoint system (10) with the adjoint source
$\mathbf{g}_{\mathbf{H}}$ calculated in step (b), and obtain the adjoint variable vector $\lambda$.

(d) Substitute $\mathbf{H}$ and $\lambda$ into (11) and calculate the sensitivity vector $d \bar{O} / d \mathbf{p}$.

\subsection{Self-adjoint formulation for inverse problems}

In this section, based on the general material sensitivity formulation of section 2.1, the self-adjoint formulation for the material sensitivity is derived for the specific form of the objective function used in the inverse problems. The aim of the inverse problem can be defined by setting the function $\mathrm{g}(\mathbf{H})$ in (1) as

$$
g(\mathbf{H})=\left|S_{11}(\mathbf{H})-S_{0}\right|^{2}
$$

where $S_{0}$ is the target value of the $S$-parameter. In case of real inverse problems, $S_{0}$ can be obtained by measurements from the actual model. For numerical inverse problems, $S_{0}$ can be obtained by calculating the numerical target model. Using the chain rule, the adjoint source $\mathbf{g}_{\mathbf{H}} \equiv \partial g / \partial \mathbf{H}$ for the adjoint system (10) is then given by,

$$
\mathbf{g}_{\mathbf{H}}=\frac{\partial g}{\partial \mathbf{H}}=\frac{\partial g}{\partial S_{11}} \frac{\partial S_{11}}{\partial \mathbf{H}}=2\left(S_{11}-S_{0}\right) \frac{\partial S_{11}}{\partial \mathbf{H}} \text {. }
$$

where $\partial S_{11} / \partial \mathbf{H}$ can be calculated from (1).

From (13), the adjoint source vector $\mathbf{g}_{\mathbf{H}}$ can be assembled by multiplying the primary source vector $\mathbf{V}$ by a constant coefficient because $S_{11}$ is a linear function of $\mathbf{H}$ on $\Gamma_{1}$, as mentioned in section 2.1. Therefore, from the linearity of the system equation, the adjoint field $\lambda$ can be calculated directly without needing to solve the adjoint system by dividing the primary solution vector $\mathbf{H}$ by the magnitude of $\mathbf{V}$, then multiplying the result by the magnitude of $\mathbf{g}_{\mathbf{H}}$.

Summarizing the above formulation, the procedure to calculate the self-adjoint material sensitivity is as follows:

(a) Solve the primary system and obtain the field solution vector $\mathbf{H}$.

(b) For a given goal function (12), calculate the adjoint source term $\mathbf{g}_{\mathbf{H}}$ using (13).

(c) Obtain the adjoint field $\lambda$ by multiplying $\mathbf{H}$ by $\left|\mathbf{g}_{\mathbf{H}} / / \mathbf{V}\right|$.

(d) Calculate the sensitivity vector $d \bar{O} / d \mathbf{p}$ using (11).

From the above procedures, the material sensitivity can be calculated using only small overhead codes dealing with steps (b) (d). For step (a), commercial EM simulators and custom solvers can be used because only the primary solution $\mathbf{H}$ is needed for the sensitivity calculation, and no differentiation of the primary system matrix is necessary. Because the adjoint variable vector $\lambda$ can be obtained directly from $\mathbf{H}$ in step (c), the computation time for sensitivity can be decreased compared to the general 
material sensitivity calculation steps shown in section 2.1 .

\section{Numerical Example}

The proposed method was applied to the several reconstruction problems of the dielectric material distribution inside a parallel plate waveguide.

\subsection{Description of the numerical models}

The design region was located $30 \mathrm{~mm}$ away from the left and right ports to allow for sufficient homogeneous waveguide regions near ports 1 and 2 . The $S$-parameter target value $S_{0}$ was calculated at 27 frequency points between $0.3 \sim 3 \mathrm{GHz}$ with frequency gap of $0.3 \mathrm{GHz}$. Four numerical models shown in Fig. 2a $\sim 5$ a were tested (model $1 \sim$ model 4). Each model was solved with two different grids. Grid 1 (coarse grid) has a $2 \mathrm{~mm} \times 2 \mathrm{~mm}$ resolution, and the total number of design cells is $52 \times 16=832$. Grid 2 (fine grid) has a $1 \mathrm{~mm} \times 1 \mathrm{~mm}$ resolution, and 3328 total cells. Note that the use of the stochastic method will be very time-consuming for grid 2 . The design variable is the normalized material density in each unit cell, which in turn controls the permittivity in that particular cell. In this paper, a modified version of the density method was used to define the relationship between the relative permittivity $\varepsilon_{r}$ and design parameter [3]:

$$
\varepsilon_{r}=\varepsilon_{\min }\left(\varepsilon_{\max } / \varepsilon_{\min }\right)^{p_{i}}
$$

where $p_{i}$ is the normalized density of the $i$ th unit cell $\left(0 \leq p_{i} \leq 1\right)$, and $\varepsilon_{\min }$ and $\varepsilon_{\max }$ are the minimum and maximum value of the relative permittivity distribution

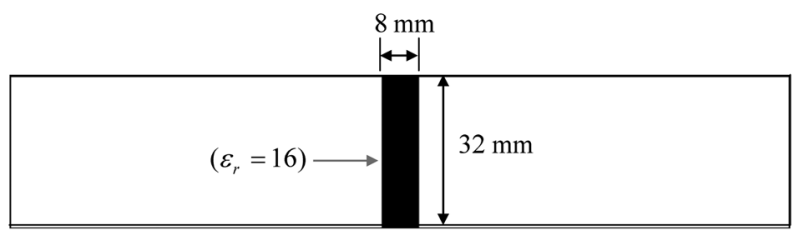

(a)

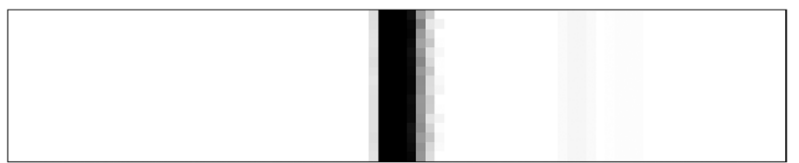

(b)

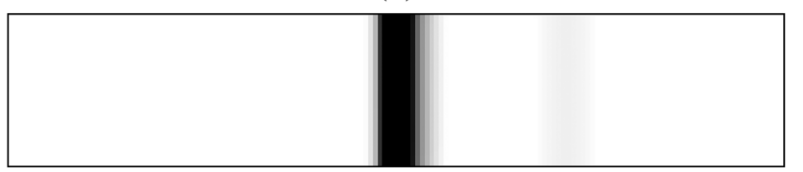

(c)

Fig. 2. Dielectric distribution for model 1. (a) Target. (b) Reconstructed (grid 1, 500 iterations). (c) Reconstructed (grid 2, 500 iterations). available in the design region, respectively.

\subsection{Results and discussion}

Figs. 2 5 show the reconstructed dielectric distribution. For models 1 and 2, a precise reconstruction of the target distribution was achieved for grids 1 and 2. For models 3 and 4 , the algorithm found the approximate location of the dielectric but the precise boundary or detailed dielectric distribution of the target model could not be replicated. In addition, different distributions were obtain-

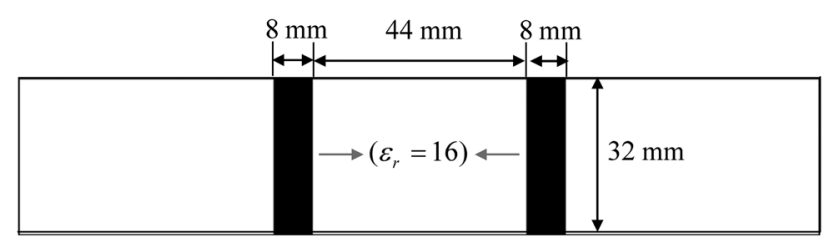

(a)

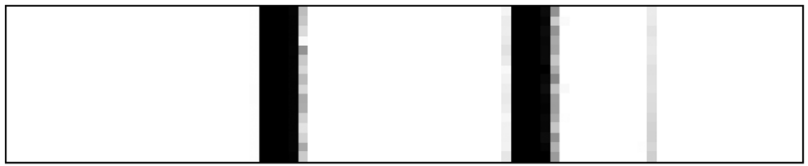

(b)

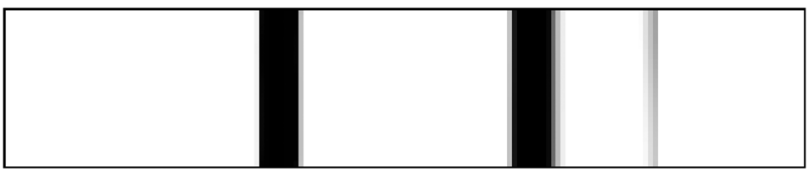

(c)

Fig. 3. Dielectric distribution for model 2. (a) Target. (b) Reconstructed (grid 1, 2000 iterations). (c) Reconstructed (grid 2, 2000 iterations).

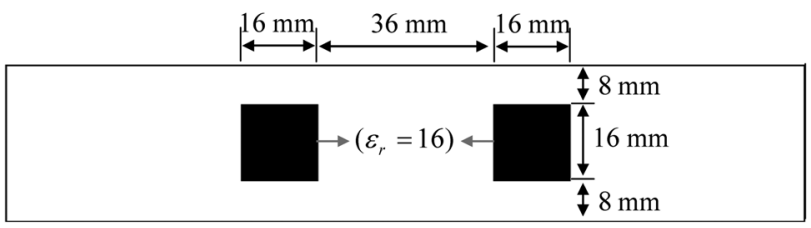

(a)

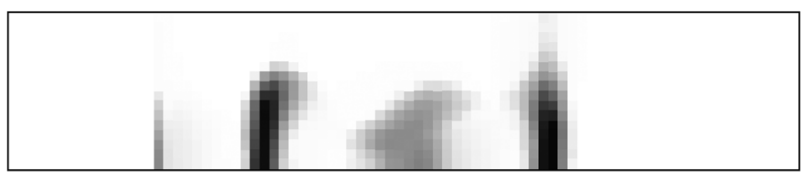

(b)

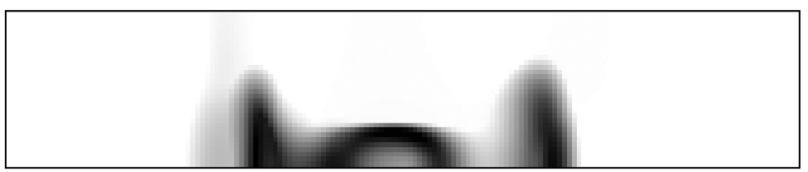

(c)

Fig. 4. Dielectric distribution for model 3. (a) Target. (b) Reconstructed (grid 1, 1000 iterations). (c) Reconstructed (grid 2, 1000 iterations). 


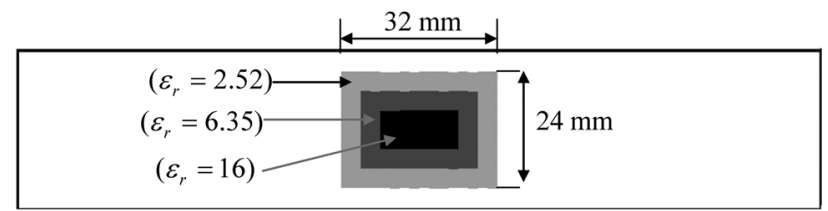

(a)

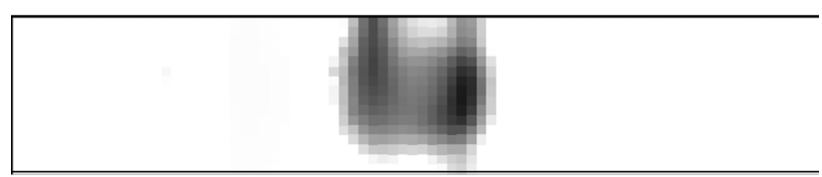

(b)

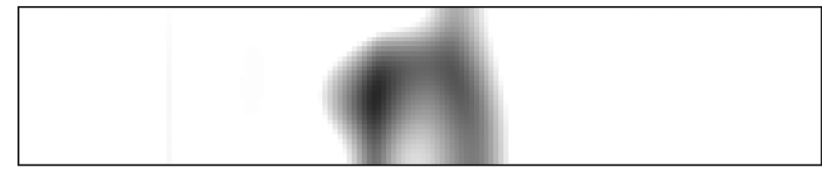

(c)

Fig. 5. Dielectric distribution for model 4. (a) Target. (b) Reconstructed (grid 1, 600 iterations). (c) Reconstructed (grid 2, 600 iterations).

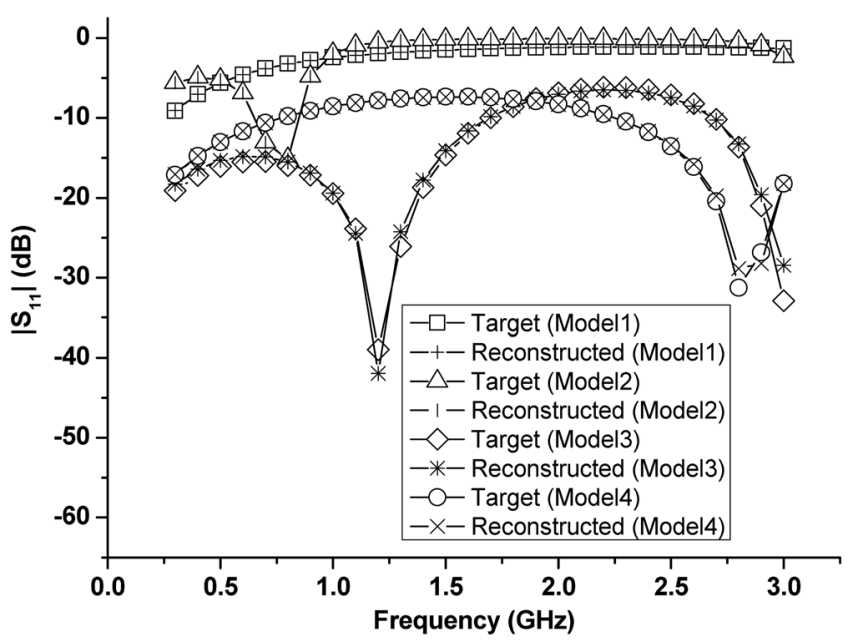

Fig. 6. $S_{11}$ magnitude plot of the target and reconstructed models.

ed from grids 1 and 2 . However, the $S$-parameter plot (Fig. 6) shows that there is good agreement between the $S$-parameter pattern of the target distribution and the reconstructed one even for models 3 and 4. To improve the result, following suggestions are given:

(a) From Fig. 2 and 3b, the regions close to port 1 have a relatively good reconstruction. However, the ones far away from port 1 have poor results. This situation can be improved by including the $S_{21}, S_{22}$, and $S_{12}$ terms in the goal function,

(b) Although higher modes of reflected and transmitted fields are considered in the analysis of the primary system, only the dominant mode of the incident field is consider-

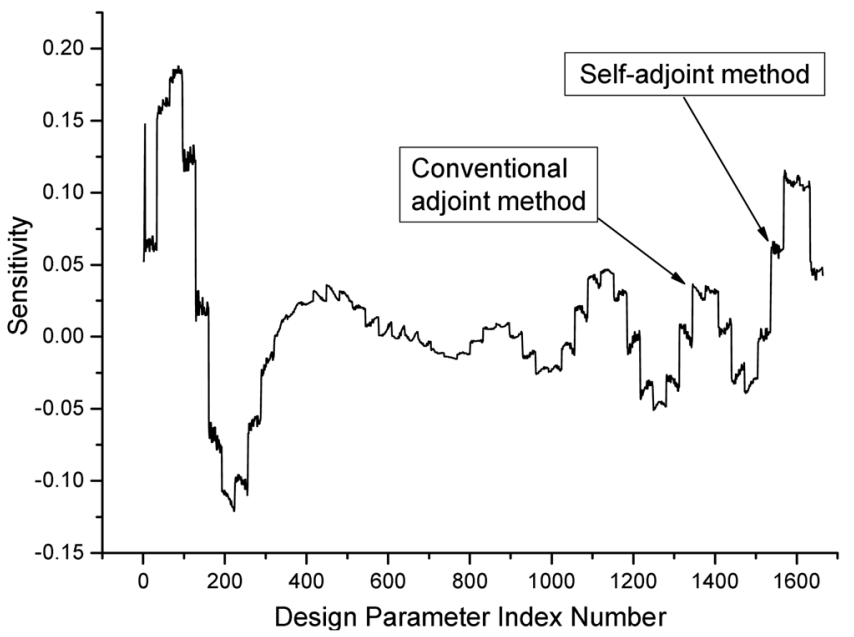

Fig. 7. Comparison of the sensitivity obtained by the selfadjoint and conventional adjoint method for model 4, grid 1, at the first iteration. The $x$-axis represents the index number of each finite element. Two finite elements form one design cell.

ed at port 1. For discontinuities like model 3 and 4, which do not span the entire height of the waveguide, this will provide insufficient information about those discontinuities. The higher mode incident fields, as well as the dominant mode ones, will provide more information on those discontinuities from the reflected and transmitted fields at the port.

The sensitivity vectors $d \bar{O} / d \mathbf{p}$ calculated using the proposed self-adjoint method agree well with those calculated using the conventional adjoint method that solves an additional adjoint equation. In other words, all components of the sensitivity vectors obtained from the two methods match precisely up to 7 significant figures, as

Table 1. Comparison of the computation time.

\begin{tabular}{ccccc}
\hline \hline Model & Grid & $\begin{array}{c}\text { Self-adjoint } \\
\text { sensitivity (sec) } \\
\text { (does not solve } \\
\text { adjoint system) }\end{array}$ & $\begin{array}{c}\text { Conventional adjoint } \\
\text { sensitivity (sec) } \\
\text { (solve adjoint system) }\end{array}$ & $\begin{array}{c}\text { Decrease in } \\
\text { computation } \\
\text { time (\%) }\end{array}$ \\
\hline \multirow{2}{*}{1} & 1 & 232.82 & 262.19 & 11.20 \\
& 2 & 1215.15 & 1342.19 & 9.47 \\
\hline \multirow{2}{*}{2} & 1 & 233.28 & 262.66 & 11.19 \\
& 2 & 1217.19 & 1340.31 & 9.19 \\
\hline \multirow{2}{*}{3} & 1 & 232.81 & 262.19 & 11.21 \\
& 2 & 1214.53 & 1336.41 & 9.12 \\
\hline \multirow{2}{*}{4} & 1 & 234.68 & 277.03 & 15.28 \\
& 2 & 1222.34 & 1341.56 & 8.89 \\
\hline
\end{tabular}

$\overline{\text { Intel Core(TM)2 Duo CPU @3.00 GHz with 2.00 GB RAM was used }}$ for the calculation. Computation time is the time required to reach 100 iterations for each model and grid. 
shown in Fig. 7.

Table 1 compares the computation time of the selfadjoint method and conventional adjoint method. The proposed method showed an approximate $10 \%$ decrease in computation time because it does not solve an additional adjoint system. However, the decrease is $<50 \%$ because the direct custom solver with a LU decomposition was used for the conventional adjoint method, which requires only forward and backward substitution to obtain the solution of the adjoint system. If commercial EM simulators without the LU decomposition technique are used, it is expected that the efficiency of the selfadjoint method will increase even further.

\section{Conclusion}

This paper proposed a novel self-adjoint material sensitivity formulation for a high frequency electromagnetic system that is independent of the specific analysis method or gridding technique. The proposed method saves approximately $10 \%$ of the total computation time by skipping the adjoint system analysis, while providing the same sensitivity information as the conventional adjoint method. Further formulations of the $S$-parameter sensitivity with respect to the PEC boundary interface and material interface utilizing a commercial EM simulator that can be applied to the wide range of high frequency optimization and inverse problems are currently underway.

\section{Acknowledgment}

This study was supported by Basic Science Research
Program through the National Research Foundation of Korea (NRF) funded by the Ministry of Education, Science and Technology (2010-0028111).

\section{References}

[1] I.-H. Park, B.-T. Lee, and S.-Y. Hahn, IEEE Trans. Magn. 28, 1533 (1992).

[2] J.-K. Byun, S.-Y. Hahn, and I.-H. Park, IEEE Trans. Magn. 35, 3718 (1999).

[3] J.-K. Byun, J.-H. Lee, I.-H. Park, H.-B. Lee, K. Choi, and S.-Y. Hahn, IEEE Trans. Magn. 36, 1144 (2000).

[4] D.-H. Kim, S.-H. Lee, I.-H. Park, and J.-H. Lee, IEEE Trans. Magn. 38, 1125 (2002).

[5] W.-C. Weng, F. Yang, and A. Z. Elsherbeni, IEEE Trans. Antennas Propag. 55, 723 (2007).

[6] A. K. Bhattacharyya, IEEE Trans. Antennas Propag. 55, 675 (2007).

[7] S. Genovesi, R. Mittra, A. Monorchio, and G. Manara, IEEE Antennas Wirel. Propag. Lett. 5, 277 (2006).

[8] J.-K. Byun and I.-H. Park, IEEE Trans. Magn. 43, 1573 (2007).

[9] J.-K. Byun, J.-H. Ko, H.-B. Lee, J.-S. Park, and H.-S. Kim, IEEE Trans. Magn. 45, 1462 (2009).

[10] N. K. Nikolova, H. W. Tam, and M. H. Bakr, IEEE Trans. Microw. Theory Tech. 52, 1207 (2004).

[11] N. K. Nikolova, X. Zhu, Y. Song, A. Hasib, and M. H. Bakr, IEEE Trans. Microw. Theory Tech. 57, 1526 (2009).

[12] D. M. Pozar, Microwave Engineering, Wiley, New York, NY (1998) pp. 196-206.

[13] J. Jin, The Finite Element Method in Electromagnetics, Wiley, New York, NY (2002) pp. 192-193. 